\title{
LIGHT-CONE EFFECT OF RADIATION FIELDS IN COSMOLOGICAL Radiative Transfer Simulations
}

\author{
KYUngJin Ahn \\ Department of Earth Sciences, Chosun University, Dong-Gu, Gwangju 501-759, Korea; kjahn@chosun.ac.kr \\ Received Jauuary 19, 2015; accepted Jauuary 23, 2015
}

\begin{abstract}
We present a novel method to implement time-delayed propagation of radiation fields in cosmological radiative transfer simulations. Time-delayed propagation of radiation fields requires construction of retarded-time fields by tracking the location and lifetime of radiation sources along the corresponding light-cones. Cosmological radiative transfer simulations have, until now, ignored this "light-cone effect" or implemented ray-tracing methods that are computationally demanding. We show that radiative transfer calculation of the time-delayed fields can be easily achieved in numerical simulations when periodic boundary conditions are used, by calculating the time-discretized retarded-time Green's function using the Fast Fourier Transform (FFT) method and convolving it with the source distribution. We also present a direct application of this method to the long-range radiation field of Lyman-Werner band photons, which is important in the high-redshift astrophysics with first stars.
\end{abstract}

Key words: cosmology: diffuse radiation — methods: numerical

\section{INTRODUCTION}

Cosmological radiative transfer simulations are used to study astrophysical processes which occur on a large, cosmic scale. A notable example of such processes is the process of cosmic reionization, in which individual H II regions take up large volumes, of order $(\sim 20$ comoving $\mathrm{Mpc})^{3}$ or larger, after $\sim 50 \%$ of all the baryons are reionized by astrophysical radiation sources (Furlanetto \& Oh 2005; Furlanetto et al. 2006; Iliev et al. 2007; Zahn et al. 2007). The typical radiation sources long after the recombination epoch are stars and quasars, which emit predominantly ultra-violet (UV) and X-ray photons, respectively. The mean free path of hydrogenionizing (H-ionizing) UV photons is simply the average size of $\mathrm{H}$ II regions, ${ }^{\mathbf{1}}$ while the mean free path of $\mathrm{X}$ ray photons is much larger than that of UV photons due to the relatively small optical depth (the scattering cross section of UV photons is much larger than that of X-ray photons: e.g., Mesinger et al. 2013; Xu et al. 2014). Therefore, the mean free path of X-ray photons is truly cosmological, surpassing several hundred $\mathrm{Mpc}$ when the rest-frame photon energy is larger than a few $\mathrm{keV}$ (Xu et al. 2014). Another example of long mean free paths is the Lyman-Werner (LW) band photons. These photons lie at the energy range of $11-13.6$ $\mathrm{eV}$, and thus traverse cosmological distances freely until they redshift into hydrogen Lyman resonance lines and are scattered off neutral hydrogen atoms (Haiman et al.

\footnotetext{
Corresponding AUTHor: K. Ahn

${ }^{1}$ Note that even after the intergalactic medium (IGM) is fully ionized, which is believed to occur at $z \lesssim 7$, the mean free path of UV photons is severely restricted to a few comoving Mpc. This is due to the rapid increase of the number of Lyman limit systems, which contain neutral hydrogen atoms inside and thus consume hydrogen-ionizing UV photons (e.g., McQuinn et al. 2011; Alvarez \& Abel 2012).
}

2000; Ahn et al. 2009). Practically all LW band photons are scattered multiple times and reprocessed into lower-energy photons when they traverse the "LymanWerner horizon" $r_{\text {LW }} \sim 100[21 /(1+z)]^{0.5} \mathrm{Mpc}$, where $z$ is the redshift at which the photons are emitted (Ahn et al. 2009).

The light-cone effect, which denotes the effect related to fields travelling at the speed of light and thus tracing past-time events along the corresponding light cones, is inherent in any physical processes on cosmic scales. Radiation fields and gravitational fields are the obvious examples governed by the light-cone effect: both fields travel at the speed of light (see Hwang et al. 2008 showing that the gravitational field travels at the speed of light in the form of the electric part of the Weyl tensor). The relevant time or length scale over which the light-cone effect matters can be estimated by an effective time-scale $t_{\mathrm{lc}} \equiv f /(d f / d t)$, where $f$ is any physical quantity relevant to the problem of interest and $d f / d t$ is the change rate of $f$. For astrophysical problems, the time scale may be naively estimated by the lifetime of radiation sources of interest. In contrast, gravitational fields under non-relativistic motion (velocity $\ll c$ ) will be corrected from the Newtonian field, which is constructed in an action-at-a-distance manner, only very slightly (about $10^{-6}-10^{-4}$ or somewhat larger if cumulative effects are considered) by general relativistic effects including this light-cone effect (Hwang et al. 2008).

Light-cone effect of radiation fields traversing cosmological distances has been usually approximated by a uniform, spatially-averaged quantity in semi-analytical studies (e.g., Haiman et al. 2000 for LW and H-ionizing backgrounds), or calculated by computationally expensive, brute-force methods of ray-tracing with finite speed of light (e.g., Bryan et al. 2014 for H-ionizing 
background). The first account of the inhomogeneous LW background in numerical simulations was taken by Ahn et al. (2009), whose consistency with semianalytical and semi-numerical approach was confirmed elsewhere (Dijkstra et al. 2008; Holzbauer \& Furlanetto 2012). The method of Ahn et al. (2009) was later used in simulating the process of cosmic reionization under the influence of the first stars (Ahn et al. 2012; Fialkov et al. 2013). This method has been improved for faster computation using FFT. Its application to other radiation fields affected by the light-cone effect is straightforward, as long as the field strength from the source is only a function of distance but not of direction. Even though this improved method was already used in the simulation by Ahn et al. (2012), its formalism and generality have never been described explicitly. Thus we lay out the generic formalism, a numerical scheme and the actual application in this paper. A similar account for X-ray background (Xu et al. 2014) used the same machinery of Ahn et al. (2009) with modifications to implement the opacity due to hydrogen and helium atoms.

This paper is organized as follows. In Section 2, we describe the generic formalism and the numerical scheme. In Section 3, we show how the LW background is calculated using this scheme, and also quantify the possible error from ignoring the light-cone effect. In Section 4, we conclude our findings and discuss further prospects and limitations.

\section{RETARDED RADiATION Field: FormalisM AND NUMERICAL METHOD}

\subsection{Green's Function Formalism}

A generic radiation field $F(\mathbf{x}, t)$ at position $\mathbf{x}$ and time $t$ in a homogeneous and isotropic background spacetime satisfies a d'Alembertian-type equation. In the trivial case of a Minkowski background, electromagnetic (EM) waves (see Jackson 1998) follow

$$
\left(\nabla_{\mathbf{x}}^{2}-\frac{1}{c^{2}} \frac{\partial^{2}}{\partial t^{2}}\right) F(\mathbf{x}, t)=-4 \pi f(\mathbf{x}, t),
$$

where $F(\mathbf{x}, t)$ is either the scalar potential $\Phi$ or the vector potential $\mathbf{A}$, and $f(\mathbf{x}, t)$ is the corresponding source term (the charge density or the current density, respectively). In this case, $F(\mathbf{x}, t)$ can be cast into an convoluted integral form

$$
F(\mathbf{x}, t)=\int d^{3} x^{\prime} d t^{\prime} G\left(\mathbf{x}, t ; \mathbf{x}^{\prime}, t^{\prime}\right) f\left(\mathbf{x}^{\prime}, t^{\prime}\right),
$$

where the retarded Green's function ${ }^{2}$ (RGF henceforth) $G\left(\mathbf{x}, t ; \mathbf{x}^{\prime}, t^{\prime}\right)$ satisfies

$$
\left(\nabla_{\mathbf{x}}^{2}-\frac{1}{c^{2}} \frac{\partial^{2}}{\partial t^{2}}\right) G\left(\mathbf{x}, t ; \mathbf{x}^{\prime}, t^{\prime}\right)=-4 \pi \delta\left(\mathbf{x}-\mathbf{x}^{\prime}\right) \delta\left(t-t^{\prime}\right)
$$

\footnotetext{
${ }^{2}$ We take the simple stance that the initial condition is well established in astrophysical problems, and therefore the retarded Green's function is more adequate than the advanced Green's function.
}

and is given in a closed form as

$$
G\left(\mathbf{x}, t ; \mathbf{x}^{\prime}, t^{\prime}\right)=\frac{\delta\left(t^{\prime}-\left[t-\frac{\left|\mathbf{x}-\mathbf{x}^{\prime}\right|}{c}\right]\right)}{\left|\mathbf{x}-\mathbf{x}^{\prime}\right|}
$$

Note that Equation (2) is in the most generic form. The light-cone effect or causality is apparent in Equations (2) and (4), as the field at observing time $t$ is caused by a source along the light cone at past time $t^{\prime}=t-\left|\mathbf{x}-\mathbf{x}^{\prime}\right| / c$. Even though Equations (1), (3) and (4) are applicable both to $\Phi$ and $\mathbf{A}$ only in the Lorentz gauge, it is well known that the electromagnetic field, as a physical quantity derived from both $\Phi$ and $\mathbf{A}$, still conserves this causality under any gauge choice (Jackson 2002; see also a nice example of Problem 6.20 in Jackson 1998).

Let us consider a generic field $F(\mathbf{x}, \tau)$ in the spacetime described by the Robertson-Walker metric with null curvature,

$$
d s^{2}=c^{2} d t^{2}-a^{2}(t) d \mathbf{x}^{2}=a(\tau)\left(d \tau^{2}-d \mathbf{x}^{2}\right)
$$

where $\mathbf{x}$ is the comoving spatial coordinate, $a(t)$ is the scale factor at conformal cosmic time $\tau$ with normalization $a=1 /(1+z)$, and $\tau$ is defined by

$$
\tau(t) \equiv \int^{t} \frac{c d t^{\prime}}{a\left(t^{\prime}\right)}
$$

It is convenient to use $\tau$ and $\mathbf{x}$ because the null geodesic is simply given by $d|\mathbf{x}| / d \tau=1$. One can then expect that the generic RGF will take the following form:

$$
\begin{aligned}
G\left(\mathbf{x}, \tau ; \mathbf{x}^{\prime}, \tau^{\prime}\right)= & \delta\left(\tau^{\prime}-\left[\tau-\left|\mathbf{x}-\mathbf{x}^{\prime}\right|\right]\right) \\
& \times \mathcal{F}\left(\left|\mathbf{x}-\mathbf{x}^{\prime}\right|, \tau, \tau^{\prime}\right),
\end{aligned}
$$

where $\mathcal{F}\left(\left|\mathbf{x}-\mathbf{x}^{\prime}\right|, \tau, \tau^{\prime}\right)$ is a generic, geometrical dilution factor. Note that $\mathcal{F}\left(\left|\mathbf{x}-\mathbf{x}^{\prime}\right|, \tau, \tau^{\prime}\right)$, in general, reflects cosmological effects such as photon-redshifting, and therefore depends also on $\tau$ and $\tau^{\prime}$. The corresponding integral form will become (cf., Equation 2)

$$
F(\mathbf{x}, \tau)=\int d^{3} x^{\prime} d \tau^{\prime} G\left(\mathbf{x}, \tau ; \mathbf{x}^{\prime}, \tau^{\prime}\right) f\left(\mathbf{x}^{\prime}, \tau^{\prime}\right)
$$

where $f\left(\mathbf{x}^{\prime}, \tau^{\prime}\right)$ is the source term properly defined in the $(\mathbf{x}, \tau)$ coordinate system.

We now decompose Equation (8) into discrete terms which are affected by different time slices, and also apply a periodic boundary condition to assimilate the usual simulation set-up. Equation (8) can be rewritten 
as

$$
\begin{aligned}
& F(\mathbf{x}, \tau) \\
& =\sum_{n} \int d^{3} x^{\prime} \int_{\tau_{n}}^{\tau_{n+1}} d \tau^{\prime} G\left(\mathbf{x}, \tau ; \mathbf{x}^{\prime}, \tau^{\prime}\right) f\left(\mathbf{x}^{\prime}, \tau^{\prime}\right) \\
& =\sum_{n} \int d^{3} x^{\prime} \int_{\tau_{n}}^{\tau_{n+1}} d \tau^{\prime} \delta\left(\tau^{\prime}-\left[\tau-\left|\mathbf{x}-\mathbf{x}^{\prime}\right|\right]\right) \\
& \quad \times \mathcal{F}\left(\left|\mathbf{x}-\mathbf{x}^{\prime}\right|, \tau, \tau^{\prime}\right) f\left(\mathbf{x}^{\prime}, \tau^{\prime}\right) \\
& \simeq \sum_{n} \int d^{3} x^{\prime} W\left(\frac{\left|\mathbf{x}-\mathbf{x}^{\prime}\right|-\left(\tau-\tau_{n+1}\right)}{\tau_{n+1}-\tau_{n}}\right) \\
& \quad \times \mathcal{F}\left(\left|\mathbf{x}-\mathbf{x}^{\prime}\right|, \tau, \tau^{\prime}\right) f\left(\mathbf{x}^{\prime}, \tau_{n}\right) \\
& \equiv \sum_{n} \int d^{3} x^{\prime} \mathcal{F}_{n}\left(\left|\mathbf{x}-\mathbf{x}^{\prime}\right| ; \tau\right) f\left(\mathbf{x}^{\prime}, \tau_{n}\right) \\
& \equiv \sum_{n} F_{n}(\mathbf{x}, \tau)
\end{aligned}
$$

where $f\left(\mathbf{x}^{\prime}, \tau_{n}\right)$ approximates the source term during $\tau^{\prime}=\left[\tau_{n}, \tau_{n+1}\right]$ as a fixed $3 \mathrm{D}$ quantity, $W(x)$ is a tophat window function such that

$$
W(x)= \begin{cases}1 & \text { if } 0 \leq \mathrm{x} \leq 1 \\ 0 & \text { otherwise }\end{cases}
$$

and the RGF and the partial contribution to $F(\mathbf{x}, \tau)$ from the sources during $\tau^{\prime}=\left[\tau_{n}, \tau_{n+1}\right]$ are defined as $\mathcal{F}_{n}\left(\left|\mathbf{x}-\mathbf{x}^{\prime}\right| ; \tau\right)$ and $F_{n}(\mathbf{x}, \tau)$, respectively. It is important to choose small enough time gaps such that $f\left(\mathbf{x}^{\prime}, \tau^{\prime}\right)$ does not evolve much during each time step. Now, we apply the periodic boundary condition: the cubical domain with size $L$ (volume $V \equiv L^{3}$ ) is infinitely repeated side to side and thus satisfies

$$
\begin{aligned}
& F_{n}(\mathbf{x}, \tau) \\
& =\sum_{\substack{u, v, w \\
\quad}} \int^{V} d^{3} x^{\prime} \mathcal{F}_{n}\left[\mid \mathbf{x}-\left\{\mathbf{x}^{\prime}+(u, v, w) L, \tau_{n}\right)\right. \\
& =\int^{V} d^{3} x^{\prime} \sum_{u, v, w} \mathcal{F}_{n}\left[\left|\mathbf{x}-\left\{\mathbf{x}^{\prime}+(u, v, w) L\right\}\right| ; \tau\right] \\
& \quad \times f\left(\mathbf{x}^{\prime}, \tau_{n}\right) \\
& \equiv \int^{V} d^{3} x^{\prime} \mathcal{G}_{n}\left(\mathbf{x}-\mathbf{x}^{\prime}\right) f\left(\mathbf{x}^{\prime}, \tau_{n}\right),
\end{aligned}
$$

where $u, v, w$ are integer indices running from $-\infty$ to $\infty$ along the three axes of the cubical domain, both $\mathbf{x}$ and $\mathbf{x}^{\prime}$ are confined to one domain, the second identity is guaranteed by the periodicity of the source term, and the last identity defines the "effective" RGF $\mathcal{G}_{n}(\mathbf{x}-$ $\left.\mathbf{x}^{\prime}\right)$. Note that for a given observing point $(\mathbf{x}, \tau)$ and the past time-slice at $\left[\tau_{n}, \tau_{n+1}\right]$, the $(u, v, w)$ points contributing to $\mathcal{G}_{n}\left(\mathbf{x}-\mathbf{x}^{\prime}\right)$ are only those confined in the spherical shell bounded by comoving radii $\tau-\tau_{n+1}$ and $\tau-\tau_{n}$.

We have just cast the full integral (Equation 8) for the retarded field into the summation (Equation 9) of spatially convoluted integrals (Equation 10). In the next section, we will describe how we calculate the full retarded field under a uniform Eulerian grid.

\subsection{Numerical Method}

We now develop a numerical method to calculate the retarded fields. A scheme for calculating 3D fields in numerical simulations becomes the most transparent under a uniform grid, and thus we restrict the description also to the case of a uniform grid. This choice is further justified by the fact that a retarded field originates from sources located far from the observing point, whose impact on each observing point is greatly averaged out and thus can be accurately calculated even on a uniform grid.

If we discretize relevant quantities on a uniform grid, Equation (10) turns into

$$
\begin{aligned}
F_{n}(i, j, k)= & \sum_{i^{\prime}=0}^{N-1} \sum_{j^{\prime}=0}^{N-1} \sum_{k^{\prime}=0}^{N-1} \mathcal{G}_{n}\left(i-i^{\prime}, j-j^{\prime}, k-k^{\prime}\right) \\
& \times f_{n}\left(i^{\prime}, j^{\prime}, k^{\prime}\right),
\end{aligned}
$$

where the domain is divided into cells with size $l \equiv L / N, \quad\{i, j, k\}$ and $\left\{i^{\prime}, j^{\prime}, k^{\prime}\right\}$ are integer indices for real-space positions, and we absorbed $l^{3}$ into $f_{n}\left(i^{\prime}, j^{\prime}, k^{\prime}\right)$ by letting $\int d^{3} x^{\prime} f\left(\mathbf{x}^{\prime}, \tau_{n}\right) \longmapsto$ $\sum_{i^{\prime}, j^{\prime}, k^{\prime}} f_{n}\left(i^{\prime}, j^{\prime}, k^{\prime}\right)$. If $\hat{A}(p, q, r)$ is the Fourier component of some field $A(i, j, k)$ given by

$$
\begin{aligned}
A(i, j, k)= & \frac{1}{N^{3}} \sum_{p=0}^{N-1} \sum_{q=0}^{N-1} \sum_{r=0}^{N-1} \hat{A}(p, q, r) \\
& \times \exp \left[\frac{2 \pi \mathbf{i}(i p+j q+k r)}{N}\right],
\end{aligned}
$$

where the imaginary unit $\mathbf{i}$ is shown in bold face to avoid confusion with the index $i$, then the convolution theorem gives

$$
\hat{F}_{n}(p, q, r)=\hat{\mathcal{G}_{n}}(p, q, r) \hat{f}_{n}(p, q, r),
$$

where $\{p, q, r\}$ are the indices of the Fourier-space coordinates.

The merit of Equations (11) - (13) is that the retarded field can now be calculated by FFT as long as periodicity is assumed. The algorithm then becomes straightforward, as follows:

1. For given (observing) time $\tau$, divide the lookback time into discrete time slices with $\tau^{\prime}=\left[\tau_{n}, \tau_{n+1}\right]$.

2 . For a given (past) time slice $n$, locate a unit source at the origin $\left(i^{\prime}, j^{\prime}, k^{\prime}\right)=(0,0,0)$ of the realspace domain (box), and attach identical boxes - each containing one unit source at its origin side by side. The total number of boxes to attach may be limited by the "effective horizon", beyond which the contribution to $\mathcal{G}_{n}(i, j, k)$ (or to the net $F_{n}(i, j, k)$ due to a rapid decrease of $f_{n}\left(i^{\prime}, j^{\prime}, k^{\prime}\right)$ in lookback time) becomes too weak to include. 


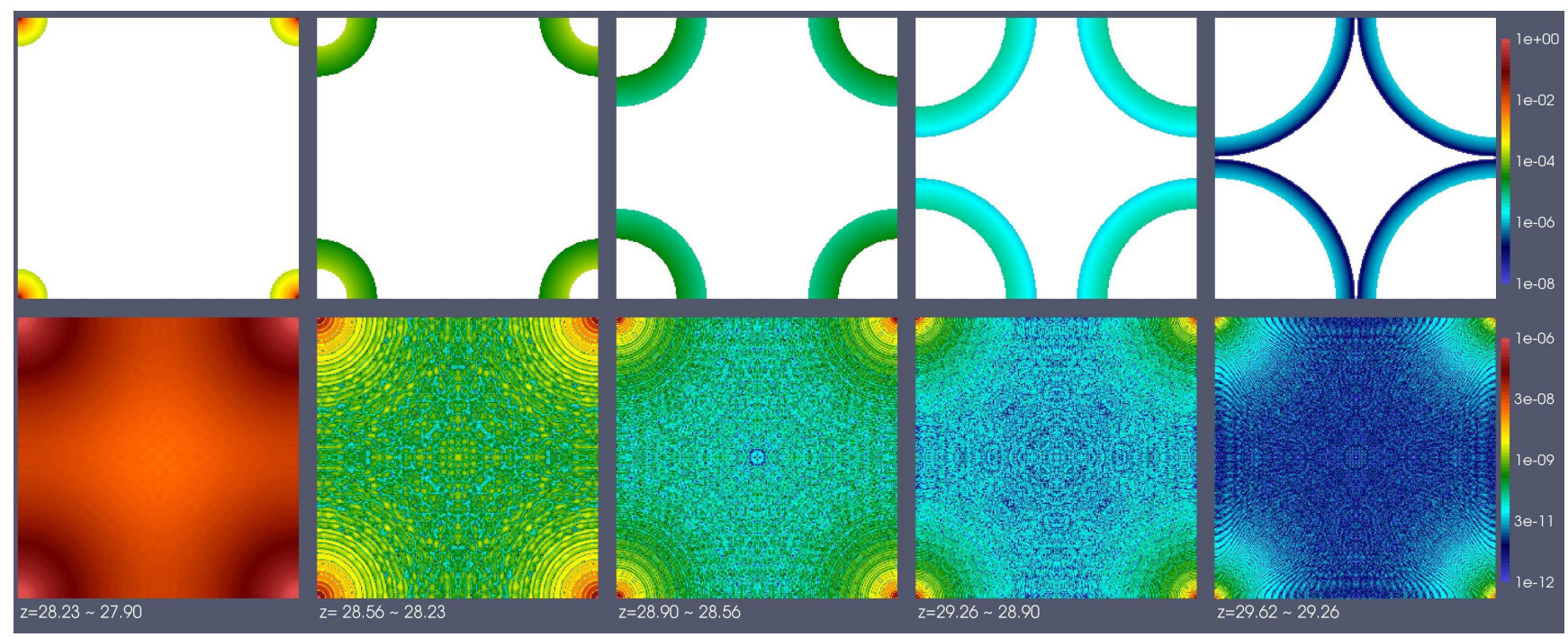

Figure 1. RGFs for LW intensity at observing redshift $z=27.90$, in a periodic box of size $114 / h$ (comoving) Mpc and a uniform grid of $256^{3}$ cells. Both the real-space (top row) and Fourier-space (bottom row) RGFs $\left(\mathcal{G}_{n}\right.$ and $\hat{\mathcal{G}_{n}}$ respectively) are shown in log scales, on a slice containing 4 corners of the box. For $\hat{\mathcal{G}_{n}}$ which has large dynamic range, we took the absolute value of its real part, $\left|\operatorname{Re}\left(\hat{\mathcal{G}_{\mathrm{n}}}\right)\right|$, for better visualization. From left to right, RGFs of progressively longer lookback-time slices are shown, in units arbitrarily chosen but consistent throughout time slices. Lookback-time range is shown in terms of redshift, below each set of $\mathcal{G}_{n}$ and $\hat{\mathcal{G}}_{n}$. Each redshift range corresponds to 2 Myrs of cosmic time. Note that the right-most panels correspond to the time slice containing the horizon $r_{\mathrm{LW}}$.

Calculate $\mathcal{G}_{n}(i, j, k)$ from this configuration by a direct summation (see its definition in Equation 10) over the contributions from these sources, when the functional form of $\mathcal{F}\left(\left|\mathbf{x}-\mathbf{x}^{\prime}\right|, \tau, \tau^{\prime}\right)$ in Equation (9) is known. Note that $\mathcal{G}_{n}(i, j, k)$ at each observing point $\{i, j, k\}$ is affected only by the unit sources inside the spherical shell given by $\tau-\tau_{n+1} \leq r_{\mathrm{os}} \leq \tau-\tau_{n}$, where $r_{\mathrm{os}}$ is the comoving distance between $\mathbf{x}=(i, j, k) l$ and $\mathbf{x}^{\prime}=(u, v, w) L$ and the index notation follows those of Equations (10) and (11).

3. Get $\hat{\mathcal{G}_{n}}(p, q, r)$ by applying FFT to $\mathcal{G}_{n}(i, j, k)$ calculated from step 2 .

4. Get $\hat{f}(p, q, r)$ by applying FFT to $f_{n}(i, j, k)$.

5 . Get $\hat{F}_{n}(p, q, r)$ from Equation (13), and then apply FFT to get $F_{n}(i, j, k)$.

6. Finally, sum over time slices to get $F(i, j, k)=$ $\sum_{n} F_{n}(i, j, k)$.

Note that once the box size, the grid resolution and the output times are determined, one can precalculate $\mathcal{G}_{n}(i, j, k)$ for each set of observing time $(\tau)$ and lookback time $\left(\tau_{n}-\tau_{n+1}\right)$ and store all $\hat{\mathcal{G}_{n}}(p, q, r)$ 's regardless of the source distribution, which is a merit of the convolution theorem. The number of data files for $\mathcal{G}_{n}(i, j, k)$ is $\sim N_{\text {observe }} N_{\text {lookback, }}$, where $N_{\text {observe }}$ is the number of observing redshifts and $N_{\text {lookback }}$ is the number of lookback times. $N_{\text {lookback }}$ can depend on the observing redshift, depending on the change of $t_{\mathrm{lc}}$ over time. When the box size $(L)$ is much smaller than the size of the effective horizon $\left(r_{h}\right)$, the number of unit sources repeated around the computation domain will be $N_{\text {unit-source }} \sim\left(r_{h} / L\right)^{3}$, and the computation of each $\mathcal{G}_{n}(i, j, k)$ will require $\sim N_{\text {mesh }}(r) N_{\text {unit-source }}$ operations.

\section{Applichtion: LyMAN-WERnER BACKGROUND}

As an application, we apply the method from Section 2.2 to the calculation of LW background. LW bands are composed of many excitation levels of hydrogen molecules $\left(\mathrm{H}_{2}\right)$, and at high intensities the LW photons can photo-dissociate $\mathrm{H}_{2}$. Because first stars are born with the help of $\mathrm{H}_{2}$-cooling, calculating $\mathrm{LW}$ background is crucial in the study of first-star formation.

The functional form of the RGF for LW background was first laid out by Ahn et al. (2009). The bandaveraged and angle-averaged intensity $J_{\mathrm{LW}}(\mathbf{x}, z)$ observed at comoving location $\mathbf{x}$ and redshift $z$ due to a source at $\left(\mathbf{x}^{\prime}, z_{\mathrm{s}}\right)$ in units of $\operatorname{erg~s}^{-1} \mathrm{~cm}^{-2} \mathrm{~Hz}^{-1} \mathrm{sr}^{-1}$ is given by

$$
J_{\mathrm{LW}}(\mathbf{x}, z)=\frac{1}{(4 \pi)^{2}} \frac{\left\langle L_{\nu}\left(\mathbf{x}^{\prime}, z_{s}\right)\right\rangle}{\left|\mathbf{x}-\mathbf{x}^{\prime}\right|^{2}} \frac{(1+z)^{3}}{1+z_{\mathrm{s}}} f_{\mathrm{mod}}\left(\left|\mathbf{x}-\mathbf{x}^{\prime}\right|\right),
$$

where $\left\langle L_{\nu}\left(\mathbf{x}^{\prime}, z_{s}\right)\right\rangle \equiv \int_{11.5 \mathrm{eV}}^{13.6 \mathrm{eV}} d\left(h_{p} \nu\right) L_{\nu}\left(\mathbf{x}^{\prime}, z_{s}\right) /(2.1 \mathrm{eV})$ is the rest-frame, frequency $(\nu)$-averaged source luminosity of the emitted LW band photons at the source redshift $z_{s}$ and $h_{p}$ is the Planck constant,

$$
f_{\text {mod }}= \begin{cases}1.7 \mathrm{e}^{-\left(\frac{\left|\mathbf{x}-\mathbf{x}^{\prime}\right|}{116.29 \alpha}\right)^{0.68}}-0.7 & \text { if } \frac{\left|\mathbf{x}-\mathbf{x}^{\prime}\right|}{\alpha} \leq 97.39 \\ 0 & \text { otherwise }\end{cases}
$$




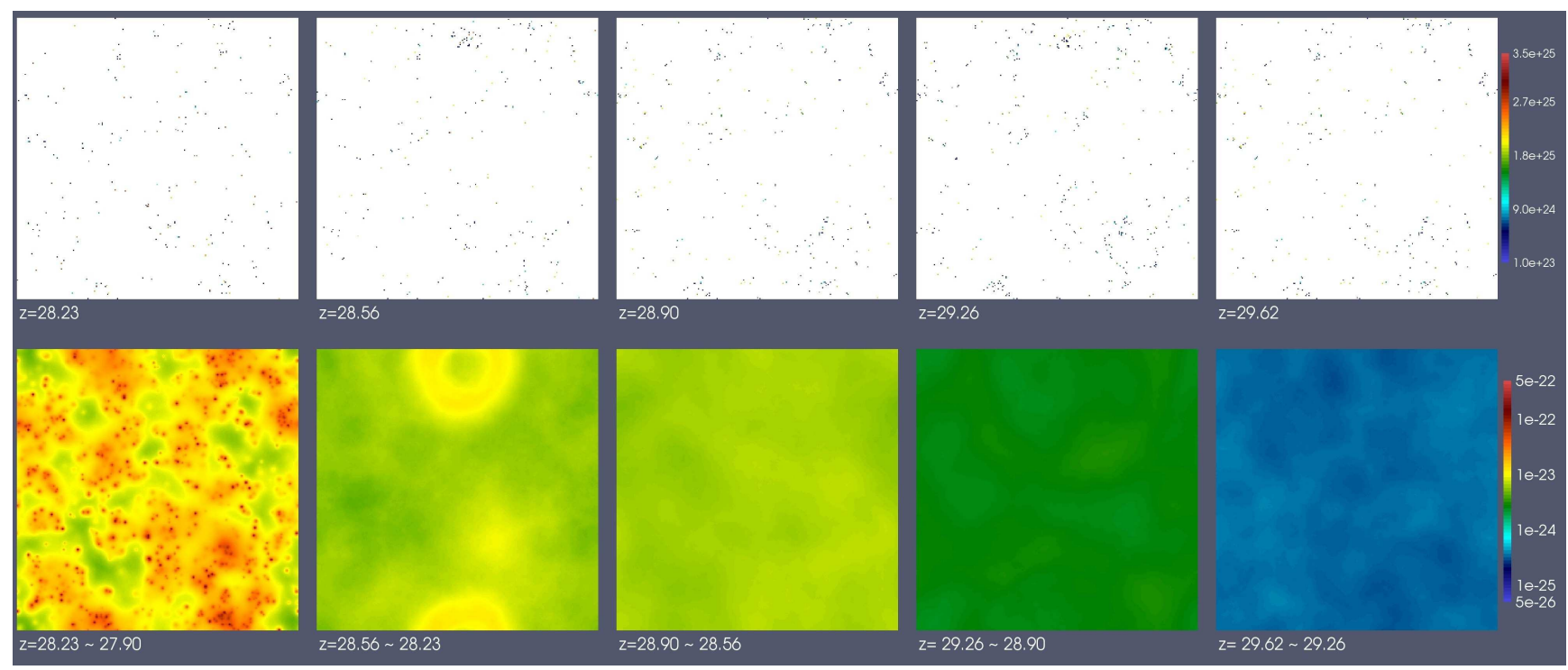

Figure 2. Source distribution and LW background from different time slices, with the same configuration as in Figure 1. The top row shows the 2D maps of $\left\langle L_{\nu}\right\rangle$ (assumed to be fixed at and beyond the redshift specified below each panel until the adjacent future redshift) on a slice of thickness $(114 / 256) / h \mathrm{Mpc}$, and the bottom row the "partial" $J_{\mathrm{LW}}$ 's on the same slice contributed from the look-back time ranges specified below each panel. The net $J_{\mathrm{LW}}$ observed at the observing redshift $z=27.90$ is given by the summation of all the partial $J_{\mathrm{LW}}$ 's, which is shown in Figure 3 .

and

$$
\alpha=\left(\frac{h}{0.7}\right)^{-1}\left(\frac{\Omega_{m}}{0.27}\right)^{-0.5}\left(\frac{1+z_{\mathrm{s}}}{21}\right)^{-0.5}
$$

with the Hubble constant (in units of $100 \mathrm{~km} / \mathrm{s} / \mathrm{Mpc}$ ) $h$ and the present ratio of matter density to the critical density $\Omega_{m}$. Of course, the light-cone effect is implicit here: $\left|\mathbf{x}-\mathbf{x}^{\prime}\right|=\tau(z)-\tau\left(z_{\mathrm{s}}\right)$. The LW horizon is $r_{\mathrm{LW}} \equiv$ $97.39 \alpha$, beyond which no sources contribute to $J_{\mathrm{LW}}$. Comparing Equation (14) to Equations (7) and (8), we find that

$$
\begin{aligned}
\mathcal{F}\left(\left|\mathbf{x}-\mathbf{x}^{\prime}\right|, \tau, \tau^{\prime}\right)= & \frac{1}{(4 \pi)^{2}\left|\mathbf{x}-\mathbf{x}^{\prime}\right|^{2}} \frac{[1+z(\tau)]^{3}}{1+z_{\mathrm{s}}\left(\tau^{\prime}\right)} \\
& \times f_{\bmod }\left(\left|\mathbf{x}-\mathbf{x}^{\prime}\right|\right),
\end{aligned}
$$

if we take the source term to be a sum of individual source luminosities such that $f\left(\mathbf{x}^{\prime}, \tau^{\prime}\right)=$ $\sum_{s}\left\langle L_{\nu}\left(\mathbf{x}_{s}^{\prime \prime}, z_{s}\left(\tau^{\prime}\right)\right)\right\rangle \delta\left(\mathbf{x}^{\prime}-\mathbf{x}_{s}^{\prime \prime}\right)$.

When visualized, the real-space $\operatorname{RGF} \mathcal{G}_{n}(i, j, k)$ can give some insight on how $J_{\mathrm{LW}}$ is constructed. Figure 1 depicts RGFs for $J_{\mathrm{LW}}$, which clearly show how the region of influence is determined by the light-cone effect from a unit source at one corner of the box ${ }^{3}$. Following the steps described in Section 2.2, we have calculated $J_{\text {LW }}$ and used this to study the formation and suppression of the first stars during the epoch of reionization (Ahn et al. 2009, 2012). Ahn et al. (2012) simulated the process of cosmic reionization including the rise and fall of first stars, in a box of size $114 / h \mathrm{Mpc}$, on which Figures $1-3$ are based. Lifetime of first stars, which are believed to be as massive as $M_{*} \gtrsim 100 M_{\odot}$ on average

\footnotetext{
${ }^{3}$ The periodic boundary condition makes unit sources appear "around" every corner of a given box, as seen in Figure 1.
}

(e.g., see a recent result by Hirano et al. 2014), is only about a few Myrs or less, and thus the life and death of these stars render the source function to change rapidly in time and space. In Figure 2, the rapid evolution of $\left\langle L_{\nu}\right\rangle$ every 2 Myrs due to first stars is shown, together with the partial contributions $F_{n}(p, q, r)$ to $J_{\mathrm{LW}} \cdot r_{\mathrm{LW}}$ resides within the lookback time of 10 Myrs. One can see that contributions to $J_{\mathrm{LW}}$ becomes weaker and more homogeneous as lookback time increases. Nevertheless, contribution of those time slices with lookback time $t_{\text {lookback }}=[2-10]$ Myrs (2nd to 5th panels from left in Figures 1 and 2) to $J_{\mathrm{LW}}$ is not completely negligible.

How important is the light-cone effect in the LW background from first stars? To answer this question, we now calculate $J_{\mathrm{LW}}$ in an action-at-a-distance way, and then compare this to $J_{\mathrm{LW}}$ correctly calculated including the light-cone effect. For this, we now freeze the source distribution at the observing redshift, while attaching identical boxes side by side for periodicity. Basically, we now calculate this "instantaneously affected" $J_{\mathrm{LW}}$ as

$$
\begin{aligned}
J_{\mathrm{LW}, \text { instant }}(\mathbf{x}, z)= & \frac{1}{(4 \pi)^{2}} \frac{\left\langle L_{\nu}\left(\mathbf{x}^{\prime}, z\right)\right\rangle}{\left|\mathbf{x}-\mathbf{x}^{\prime}\right|^{2}} \frac{(1+z)^{3}}{1+z_{\mathrm{s}}} \\
& \times f_{\text {mod }}\left(\left|\mathbf{x}-\mathbf{x}^{\prime}\right|\right),
\end{aligned}
$$

where $\left\langle L_{\nu}\right\rangle$ is now frozen at the observing redshift $z$ (e.g., the source term corresponding to the top-leftmost panel in Figure 2), but we keep all other terms intact including the implicit condition $\left|\mathbf{x}-\mathbf{x}^{\prime}\right|=\tau(z)-\tau\left(z_{\mathrm{s}}\right)$, in order to quantify only the impact of the temporal evolution of the source term. Figure 3 shows that the rapid evolution of source term indeed imprints its signature in $J_{\mathrm{LW}}$. The fractional difference $f_{\text {diff }} \equiv$ $\left(J_{\mathrm{LW}}\right.$, instant $\left.-J_{\mathrm{LW}}\right) / J_{\mathrm{LW}}$ is about $\sim 10 \%$, which is quite 


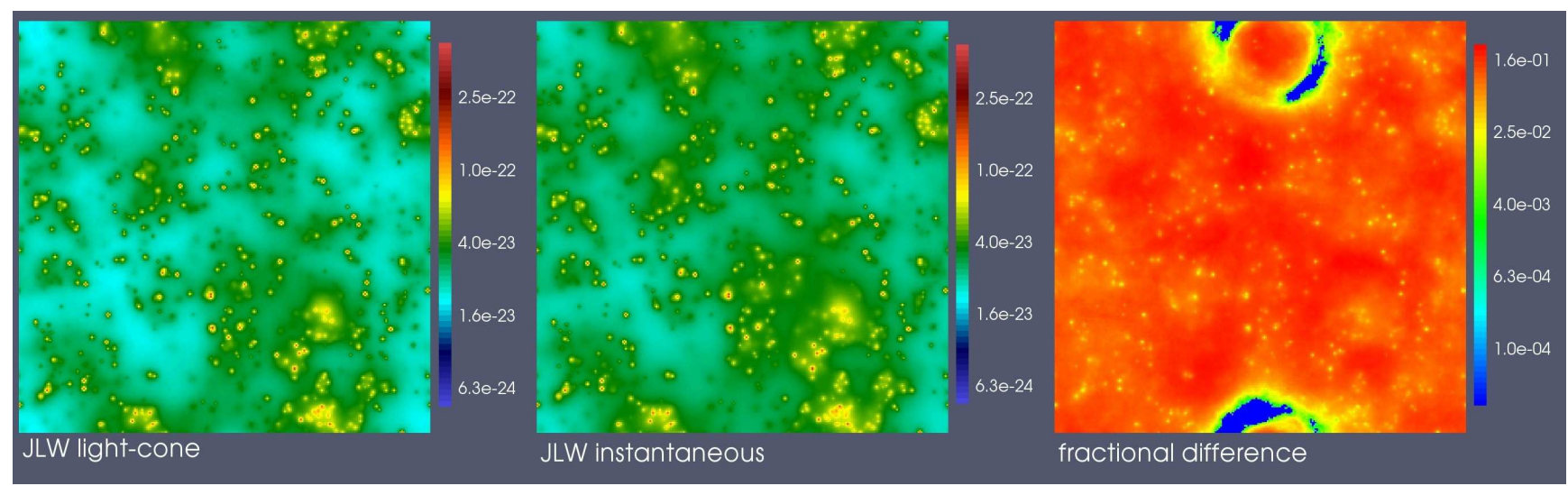

Figure 3. Comparison of $J_{\mathrm{LW}}$ 's with the light-cone effect and without. $J_{\mathrm{LW}}$ under the correctly implemented light-cone effect (left), $J_{\mathrm{LW}}$ under an action at a distance (middle; see Equation 18) and the fractional difference between these two fields (right) are plotted in the same 2D slice as in Figures 1 and 2.

significant. Therefore, astrophysical processes governed by radiation sources of short lifetime should be affected by the light-cone effect in general.

\section{Discussion}

We have formulated a generic method to numerically calculate radiation fields which are affected by the lightcone effect. This method requires discretizing both time and space coordinates, and is analogous to the usual mesh scheme used in N-body gravity simulations. The light-cone effect is realized by the retarded-time Greeen's function, and its calculation becomes efficient when a periodic-box condition is used. Once the RGFs are calculated, the process of obtaining a radiation field of interest is performed by FFT, which greatly expedites computation compared to direct summation over all sources inside and outside the simulation box.

The relevance of this method is clear in astrophysical problems occurring on cosmological scales. Once the size of the simulation box is chosen and the functional form of the RGF is known, one can follow the algorithm in Section 2.2, as in the example of LW background in Section 3. Similar large-scale astrophysical processes which require an accurate account of the light-cone effect are the reionization of baryons by UV photons and the reionization and heating of gas by X-ray photons. It becomes more important at very high redshift when most astrophysical sources are first stars and their byproducts, because the lifetime of first stars are believed to be very short and thus the source function evolves rapidly in time (Section 3 ).

There are, of course, limitations to this method. The method is based on the fact that the RGF is a function only of distance to the source but not of direction to the source. In reality, radiative transfer is usually affected by the actual path of the ray in the form of the locallyvarying optical depth. For example, formation of an $\mathrm{H}$ II bubble from a source does not occur in a spherically symmetric way, even though light travels at the speed of light. This is due to the inhomogeneous distribution of hydrogen density (and also the optical depth), which then regulates the $\mathrm{H}$-ionizing background to be dependent on the direction of the ray. For H-ionizing background, our method can then be used only after the IGM is fully ionized and the optical depth becomes negligible everywhere. In case of LW background, we could safely use the method because the modulation factor $f_{\text {mod }}$ in Equation (15), derived from the cosmic redshifting of LW photons and the frequency of hydrogen Lyman resonance lines, is valid even when the IGM is ionized, because simulations of cosmic reionization find that the IGM leaves trace amount of neutral hydrogen atoms due to recombination (e.g., Iliev et al. 2008). In this case, very-high opacity of Lyman resonance lines guarantees that once LW band photons are redshifted into one of these lines, even a trace amount of hydrogen atoms can successfully scatter off those photons, and then $f_{\bmod }$ becomes the only modulation factor in addition to the usual geometrical dilution factor (Equations 14 and 17). Another limitation is that the method cannot treat the gravitational lensing effect, which depends on the actual mass distribution around each ray's path. If a problem of interest is found to be strongly affected by the lensing effect, one should resort back to the ray-tracing method. In addition, if source locations are resolved beyond the mesh resolution, one can adaptively choose to use the particle-mesh (PM) scheme to calculate the near-source influence by direct summation over nearby-source contributions while calculating far-source influence by our method.

\section{ACKNOWLEDGMENTS}

We are grateful to the anonymous referee who gave a prompt and accurate report. This work was supported by the NRF grant funded by the Korean government MEST (NRF-2012R1A1A1014646; NRF2014R1A1A2059811).

\section{REFERENCES}

Ahn, K., Shapiro, P. R., Iliev, I. T., Mellema, G., \& Pen, U. 2009, The Inhomogeneous Background Of 
$\mathrm{H}_{2}$-Dissociating Radiation During Cosmic Reionization, ApJ, 695, 1430

Ahn, K., Iliev, I. T., Shapiro, P. R., Mellema, G., Koda, J., \& Mao, Y. 2012, Detecting the Rise and Fall of the First Stars by Their Impact on Cosmic Reionization, ApJL, 756, L16

Alvarez, M. A., \& Abel, T. 2012, The Effect of Absorption Systems on Cosmic Reionization, ApJ, 747, 126

Bryan, G. L., Norman, M. L., O'Shea, B. W., Abel, T., Wise, J. H., Turk, M. J., Reynolds, D. R., Collins, D. C., Wang, P., Skillman, S. W., Smith, B., Harkness, R. P., Bordner, J., Kim, J.-h., Kuhlen, M., Xu, H., Goldbaum, N., Hummels, C., Kritsuk, A. G., Tasker, E., Skory, S., Simpson, C. M., Hahn, O., Oishi, J. S., So, G. C., Zhao, F., Cen, R., Li, Y., \& The Enzo Collaboration. 2014, ENZO: An Adaptive Mesh Refinement Code for Astrophysics, ApJS, 211, 19

Dijkstra, M., Haiman, Z., Mesinger, A., \& Wyithe, J. S. B. 2008, Fluctuations in the High-Redshift Lyman-Werner Background: Close Halo Pairs as the Origin of Supermassive Black Holes, MNRAS, 391, 1961

Fialkov, A., Barkana, R., Visbal, E., Tseliakhovich, D., \& Hirata, C. M. 2013, The 21-cm Signature of the First Stars during the Lyman-Werner Feedback Era, MNRAS, 432, 2909

Furlanetto, S. R., McQuinn, M., \& Hernquist, L. 2006, Characteristic Scales during Reionization, MNRAS, 365, 115

Furlanetto, S. R., \& Oh, S. P. 2005, Taxing the Rich: Recombinations and Bubble Growth during Reionization, MNRAS, 363, 1031

Haiman, Z., Abel, T., \& Rees, M. J. 2000, The Radiative Feedback of the First Cosmological Objects, ApJ, 534, 11
Hirano, S., Hosokawa, T., Yoshida, N., Umeda, H., Omukai, K., Chiaki, G., \& Yorke, H. W. 2014, One Hundred First Stars: Protostellar Evolution and the Final Masses, ApJ, 781,60

Holzbauer, L. N., \& Furlanetto, S. R. 2012, Fluctuations in the High-Redshift Lyman-Werner and Ly $\alpha$ Radiation Backgrounds, MNRAS, 419, 718

Hwang, J.-C., Noh, H., \& Puetzfeld, D. 2008, Cosmological Non-Linear Hydrodynamics with Post-Newtonian Corrections, J. Cosmol. Astropart. Phys., 3, 10

Iliev, I. T., Mellema, G., Shapiro, P. R., \& Pen, U. 2007, Self-Regulated Reionization, MNRAS, 376, 534

Iliev, I. T., Shapiro, P. R., McDonald, P., Mellema, G., \& Pen, U.-L. 2008, The Effect of the Intergalactic Environment on the Observability of Ly $\alpha$ Emitters during Reionization, MNRAS, 391, 63

Jackson, J. D. 1998, Classical Electrodynamics, 3rd edn. (New York: Wiley \& Sons)

Jackson, J. D. 2002, From Lorenz to Coulomb and Other Explicit Gauge Transformations, Am. J. Phys., 70, 917

McQuinn, M., Oh, S. P., \& Faucher-Giguère, C.-A. 2011, On Lyman-Limit Systems and the Evolution of the Intergalactic Ionizing Background, ApJ, 743, 82

Mesinger, A., Ferrara, A., \& Spiegel, D. S. 2013, Signatures of X-Rays in the Early Universe, MNRAS, 431, 621

Xu, H., Ahn, K., Wise, J. H., Norman, M. L., \& O'Shea, B. W. 2014, Heating the Intergalactic Medium by X-Rays from Population III Binaries in High-Redshift Galaxies, ApJ, 791, 110

Zahn, O., Lidz, A., McQuinn, M., Dutta, S., Hernquist, L., Zaldarriaga, M., \& Furlanetto, S. R. 2007, Simulations and Analytic Calculations of Bubble Growth during Hydrogen Reionization, ApJ, 654, 12 